\title{
Iterative Clipping and Filtering Technique for PAPR Reduction in OFDM System without Encoding
}

\author{
Kapil Sahu ${ }^{1}$, Prof. R. K. Khanna ${ }^{2}$ and Prof. K.T.Veeramanju ${ }^{3}$
}

\begin{abstract}
In a 4G wireless communication systems, bandwidth is a significant factor and service provider constantly working on to accommodating more number of users within limited allocated bandwidth. Orthogonal Frequency Division Multiplexing (OFDM) is broadly used technique and originally developed from the multi carrier modulation techniques. The major drawback of the OFDM system is large Peak to Average Power Ratio (PAPR) which can overweigh all the probable benefit of the OFDM system. Due to high PAPR, the power amplifier lead to saturation and generates inter modulation among the subcarriers. In this paper, we propose iterative clipping and filtering technique without encoding technique that clipped the amplitude of the OFDM signal, which reduces more PAPR of OFDM system after 1 or 2 iteration. Whereas classical ICF method require 8 to 16 iteration for reducing the same level of PAPR reduction. MATLAB coding result shows that sharp drop on Complementary Cumulative Distribution Function (CCDF) Curve and reduced the PAPR.

Keyword-Orthogonal Frequency Division Multiplexing (OFDM), Peak-to-Average Power Ratio (PAPR), iterative clipping and filtering (ICF), Complementary Cumulative Distribution Function $(\mathrm{CCDF}))$.
\end{abstract}

\section{INTRODUCTION}

Orthogonal Frequency Division Multiplexing (OFDM) is broadly used in wireless communication systems. Because of its many benefits such as high data rate, high spectrum efficiency and less complex equalization.[1] OFDM technique used in some of the newest and most advance standard like fixed WLAN IEEE 802.11, Wi MAX IEEE 802.16 AND 4G.[1] OFDM uses multicarrier orthogonal subcarriers and summation of these subcarriers result high peak to average power ratio (PAPR) which lead to the transmit power amplifier (TPA) into saturation and non-linearity is introduced into the signal. It is the main drawback of the OFDM system. Because of its, signal is distorted and efficiency of transmit power amplifier is degrade. [3], [5] There have been many techniques proposed to reduce the PAPR of the OFDM signal such as coding, tone injection, selective mapping (SLM), tone reservation, companding, partial transmit sequences (PTS) and amplitude clipping and filtering etc. [6]

However, there are certain limitations with these techniques. Therefore, Block coding is also used for reduction of PAPR but it is not a efficient technique to reduce PAPR up to require level. PAPR reduction can be achieved with clipping and companding technique. But these techniques introduce

\footnotetext{
${ }^{1}$ Department of Electronics and Communication Vivekananda Global University, Jaipur

${ }^{2}$ Department of Electronics and Communication Vivekananda Global University, Jaipur

${ }^{3}$ Department of Electrical and Electronics Sri Jayachamarajendra College of Engineering, Mysore
} 
distortion, which results in increased BER. [10] On the converse, SLM and PTS schemes techniques need to transmission of side information (SI) which reduces the data rate. It is also require excessive amount of inverse fast fourier transform (IFFT) calculations and hence the associated complexity is very high. $[7,8]$

\section{ITERATIVE CLIPPING AND FILTERING TECHNIQUE}

Clipping ratio $(C R)$ is defined as the ratio of clipping threshold $\left(\mathrm{C}_{\mathrm{th}}\right)$ to the root mean square power level $\left(\mathrm{P}_{\mathrm{rms}}\right)$ of the OFDM signal. [3]

$$
\text { Clpping ratio }=20 \log _{10}\left(\frac{\mathrm{DLh}}{\mathrm{Prma}}\right) d \mathrm{~d}
$$

The clipping level $\left(\mathrm{D}_{\mathrm{th}}\right)$ is recalculated in each iteration as per the constant value called clipping ratio. Clipping ratio and PAPR are related as [1] :

$$
t K=\sqrt{F A F R_{\max }}
$$

Clipping ratio is also depending on modulation scheme. Amplitude Clipping is the simplest technique that reduces peak of PAPR at small cost of performance degradation The out-of-band radiation caused by high peak to average power ratio and originated from the side lobes of the modulated subcarriers. [4] This can be minimized by iterative clipping and filtering method.

In this paper, we propose a simple PAPR reduction technique based on iterative clipping and filtering without encoding. In this method, the amplitudes of the signal are clipped in a small amount according to the threshold value using iterative clipping and filtering (ICF) method without encoding to reduce the PAPR of OFDM signal. We have also compared our results with existing technique.

\section{OFDM SYSTEM AND PAPR DESCRIPTION}

An OFDM trans-receiver is shown in Figure1. Here input bit stream processed through the QAM modulator to map the input data into symbol. Serial to parallel $(\mathrm{S} / \mathrm{P})$ convertor process the input data symbol and produce a complex vector of size N. [6]

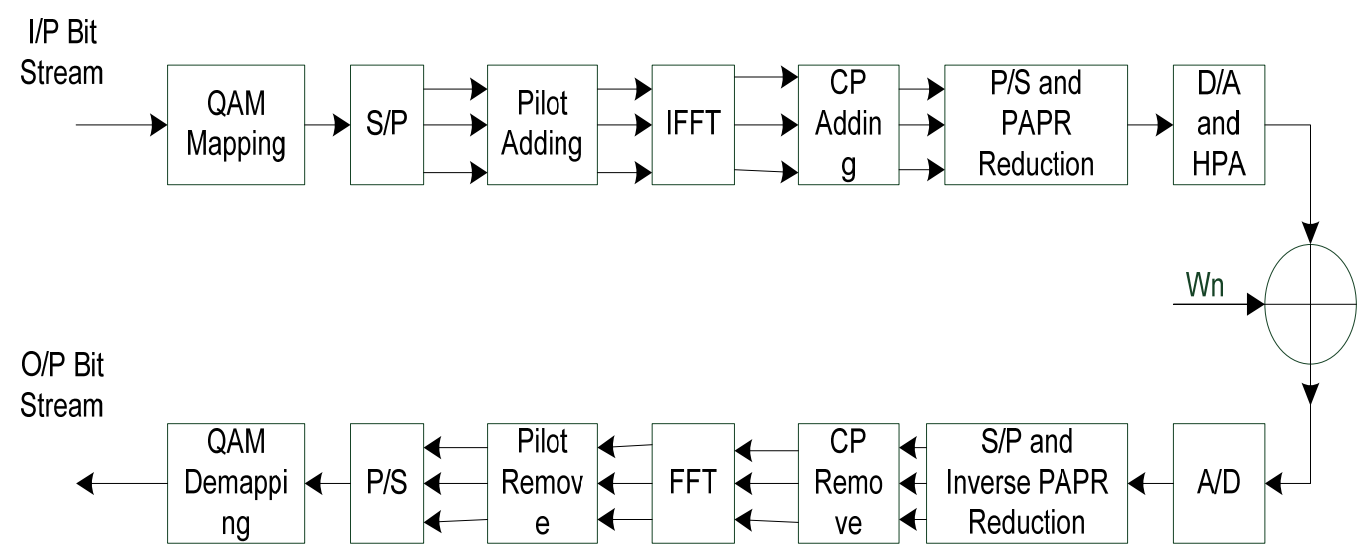

Figure 1. An OFDM system model 
Where $\mathrm{N}$ is the number of subcarrier in each OFDM symbol. Next the pilot carrier are added and converted to bit size of 64 for IFFT. These symbol are sent through the IFFT block and perform IFFT operation on these symbols and generate $\mathrm{N}$ parallel data stream.

Output signal of IFFT can be written as [11]

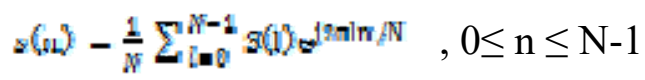

This transformed output is appended with the cyclic prefix and this added before transmission. Cyclic prefix is used to avoid the intersymbol interference and then input data symbols are passed to the parallel to serial convertor. This data passed through the Gaussian channel with sufficient input power. At the receiver, the reverse operation is performed to achieve the original data. [6]

Output signal of FFT can be written as [11]

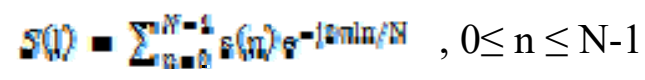

In practice, OFDM modulation and demodulation can be implemented using IFFT and FFT respectively. Iterative clipping and filtering method without encoding used to improve the PAPR of the OFDM system result efficiency of the high power amplifier improved. [4]

The amplitude $|s(n)|$ has Rayleigh distribution [1]. The PAPR of the OFDM signal in one symbol period, defined as [9]

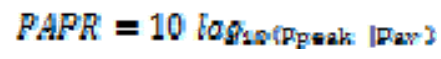

Where $\mathrm{P}_{\text {peak }}$ and $\mathrm{P}_{\mathrm{av}}$ can be compute as:

$\mathrm{P}_{\text {peak }}=\max |s(\mathrm{n})|^{2}$

$R_{Q v}=E\left[|s(n)|^{*}\right]$

Hence, the PAPR is expressed as [10]:

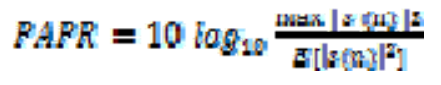

Where $|s(\mathrm{n})|^{2}$ is the peak signal power, $\mathrm{P}_{\mathrm{av}}$ average signal power and $\mathrm{E}\{$.$\} represents the expected value$ operation. In general the input and output signal of IFFT are random data.

The complementary cumulative distribution function is the distribution of PAPR that shows the amount of time a signal spends above the average power level of the measure signal. The CCDF can be given by [9]:

$$
\text { CCDF } 11-\operatorname{Pr}(R A P R \text { is Dthi) }
$$

\section{PROPOSED PAPR REDUCTION TECHNIQUE}

Here, we proposed an iterative clipping and filtering technique without encoding for PAPR reduction. This method is based on amplitude clipping and filtering [1-4]. 
Following steps involve in the proposed technique:

1. First perform QAM mapping on the input data stream.

2. Pilot carriers are used for monitoring path shift and Inter carrier interference (ICI).

3. After performing IFFT, Signal is clipped in time domain and can be expressed through this expression [4]

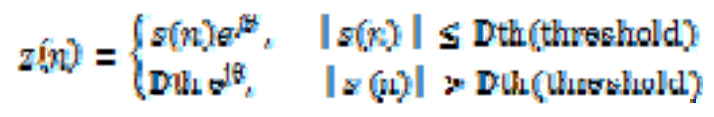

4. Cyclic prefix is added to avoid the intersymbol interference.

5. The clipped time domain signal $s(\mathrm{n})$ is converted back into the frequency domain signal by using FFT.

6. The transmitted signal is generated after FFT and PAPR of the OFDM signals are reduced by using iterative clipping.

7. Clipping ratio and number of iteration are decided on the basis of trade-off between the bit error rate and peak-to-average power ratio.

TABLE I. OFDM Parameter

\begin{tabular}{|c|c|}
\hline Parameter & Value \\
\hline Number of data subcarrier & 52 \\
\hline Number of pilot subcarrier & 12 \\
\hline Number of FFT/IFFT point & 64 \\
\hline Clipping Ratio (CR) & 1.311 \\
\hline Modulation & 8 QAM \\
\hline Channel & AWGN \\
\hline Number of data symbol & 50 \\
\hline
\end{tabular}

\section{RESULT}

In this section, we present the performance of the proposed technique on the basis of the parameter shown in table 1.

\section{A. PAPR Performance}

The performance metric used in our analysis is CCDF. Such a metric is repeatedly used performance evaluation metric. Figure 2 shows the graph between PAPR and Number of symbol. We can observe that value of PAPR vary around 1.4 and the peak value of the PAPR appears around at 5.8. 


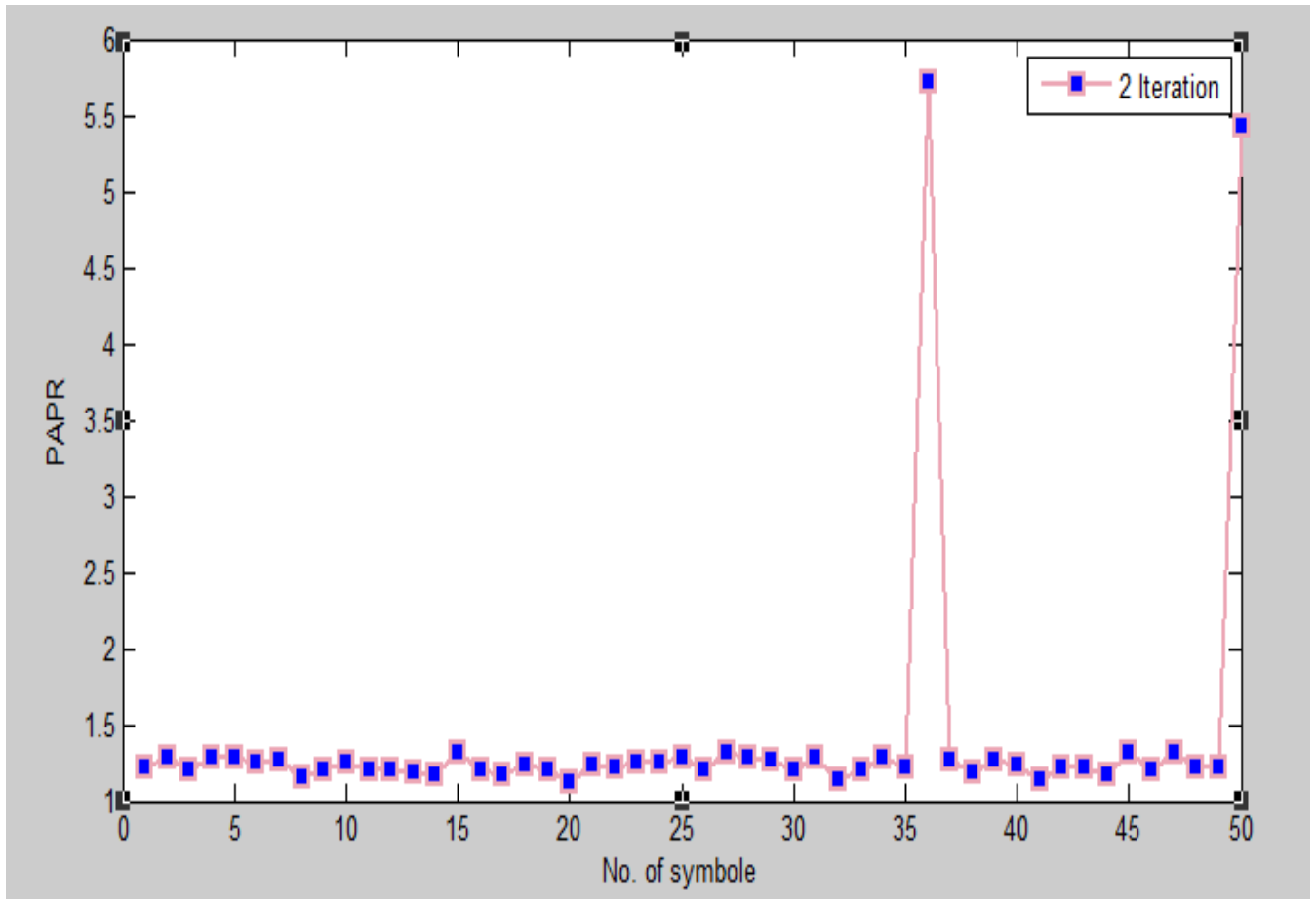

Figure 2. PAPR vs. No. of Symbol

Fig. 3 shows the graph between PAPR and CCDF. This graph shows that CCDF is sharply decreased around PAPR of 1.4 and CCDF goes to zero around 5.8 value of PAPR.

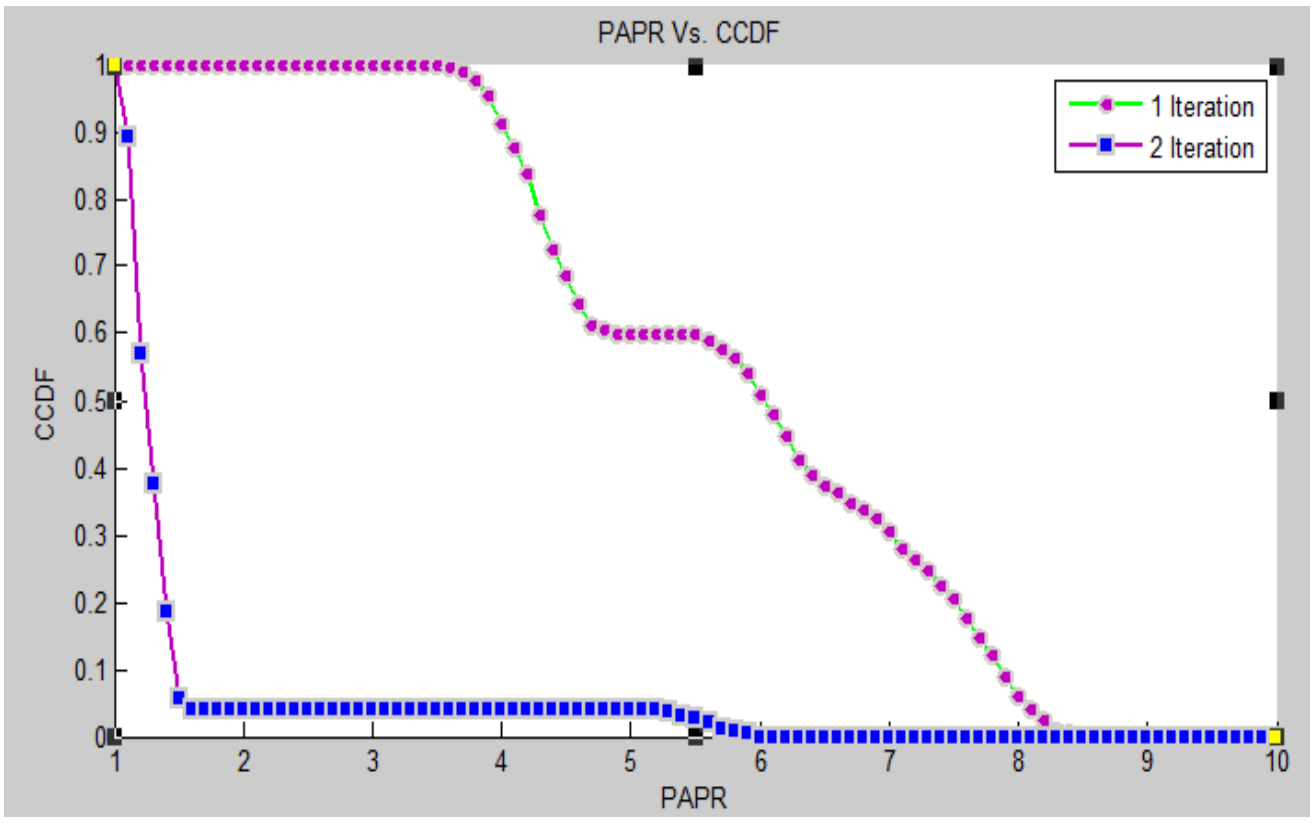

Figure 3. CCDF vs. PAPR

Optimized iterative clipping and filtering method reduce the PAPR about $5.4 \mathrm{~dB}$ after one iteration and $5.9 \mathrm{~dB}$ after two iterations. On the other hand, classical ICF method requires 8 and 16 iteration respectively to reduce the same level of PAPR. [1] But our proposed method obtain reduced PAPR 
value about $5.8691 \mathrm{~dB}$ after first iteration and $1.4019 \mathrm{~dB}$ after second iteration at a probability of approximately $10^{-1}$ as shown in figure 4 .

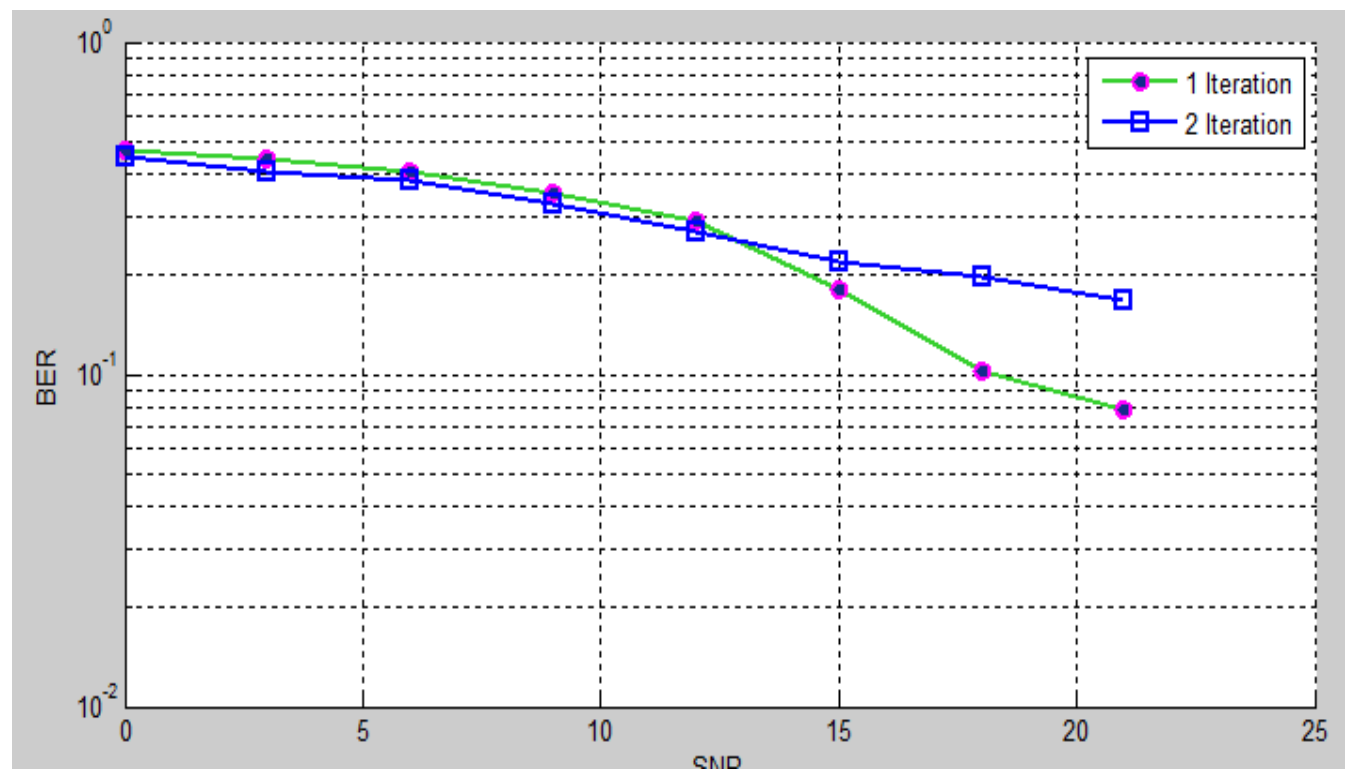

Figure 4. BER vs. SNR

The BER performance of a clipped OFDM system depends on the in-band distortion. BER performance is measured in terms of SNR which is varying from 0 to $22 \mathrm{~dB}$ as shown in Fig. 5.

\section{CONCLUSION}

In this paper we proposed a simple iterative clipping and filtering technique without encoding to reduce the PAPR of the OFDM signals. Matlab coding result shows that the proposed technique reduces PAPR more than the existing classical ICF and optimized ICF method. We can further improve the BER performance by using MIMO technique.

\section{REFERENCES}

[1] Aggarwal A., Kapadia P. and Trivedi Y.N., "PAPR Reduction in OFDM Systems: A Simple Approach Based on Clipping and Differential Scaling”, IEEE Conference Devices and Communications (ICDeCom), pp. 1-5, Feb 2011.

[2] Y. C. wang and Z. Q. Lue, "Optimized iterative clipping and filtering for PAPR reduction of OFDM system”, IEEE Transaction on Communication, vol. 59, no. 1, January 2011.

[3] Sharma, C. , Sharma, P.K. and Tomar S.K. ; Gupta, A.K., "A modified Iterative Amplitude clipping and filtering technique for PAPR reduction in OFDM systems", IEEE Emerging Trends in Networks and Computer Communications (ETNCC), pp. 365-368, April 2011. 
[4] More, A.P. and Somani S.B., "The Reduction of PAPR in OFDM Systems Using Clipping and SLM Method", IEEE Conference on Information Communication and Embedded Systems (ICICES), pp. 593-597, Feb. 2013.

[5] Zelenkov A.V. and Litvinenko A., "OFDM PAPR reduction by pre-scrambling and clipping”, IEEE Conference, Electronics Conference (BEC), 13th Biennial Baltic, pp. 141-144, Oct. 2012.

[6] Vinit V. Patel and R. N. Patil, "Minimization of PAPR in OFDM System using IDWT/DWT, Clipping and Filtering combine with Huffman Coding”, IEEE International Conference on Communication and Signal Processing, pp. 250-254, April 3-5, 2013.

[7] Shang-Kang Deng and Mao-Chao Lin, "OFDM PAPR Reduction using Clipping with Distortion control”, IEEE International Conference on Communication, vol. 4, pp. 2563-2567, 2005.

[8] Seung Hee Han and Jae Hong Lee, "An overview of peak-to-average power ratio reduction technique for multicarrier transmission”, IEEE wireless Communication, vol. 12, issue 2, pp. 5665, April 2005.

[9] Sroy Abouty, Li Renfa, Zeng Fanzi and Fall Mangone, "A Novel Iterative Clipping and Filtering Technique for PAPR reduction of OFDM signal: system using DCT/IDCT transform”, International Journal of Future Generation Communication and Networking, vol. 6, no. 1, February, 2013.

[10] Pawan Sharma, Seema Verma, "Reducing PAPR of the OFDM based wireless system using companding with convolutional codes", International journal of distributed and parallel system (IJDPS), Vol. 2, No. 6, November 2011.

[11] Alan V. Oppenheim, Ronald W. Schefer, "Digital Signal Processing”, pp. 284-287, PHI 2011. 